\title{
Efficacy of Lignocaine in Gel and Spray form during Buccal Infiltration Anesthesia in Children: A Randomized Clinical Trial
}

\author{
${ }^{1}$ Anshul Sharma, ${ }^{2}$ BS Suprabha, ${ }^{3}$ Ramya Shenoy, ${ }^{4}$ Arathi Rao
}

\section{ABSTRACT}

Aim: To compare the efficacy of $15 \%$ lignocaine spray and $8 \%$ lignocaine gel as a topical anesthetic, in reducing pain, during buccal infiltration in children.

Materials and methods: Forty-two patients aged between 7 and 12 years requiring restorative procedures/extraction/pulp therapy of primary/ permanent teeth in the maxillary arch, under buccal infiltration anesthesia were selected for the study. The participants were randomly allocated into 2 groups of 21 each. In group A, 8\% lignocaine gel and in group B, 15\% lignocaine spray was applied prior to buccal infiltration. Pain was assessed using Wong-Baker faces pain rating scale (WBFPRS) and faces legs activity cry and consolability (FLACC) painscale.

Results: Pearson's chi-square test revealed that there was no significant difference in the FLACC scores of the two groups $(p=0.54)$. Independent $t$-test demonstrated that there was no significant difference in Wong-Bakers faces pain score between the two agents $(p=0.07)$.

Conclusion: There is no significant difference in the efficacy of $15 \%$ lignocaine spray and $8 \%$ lignocaine gel as a topical anesthetic in controlling pain during buccal infiltration anesthesia, in children.

Keywords: Topical anesthesia, Lignocaine, Randomized trial.

How to cite this article: Sharma A, Suprabha BS, Shenoy R, Rao A. Efficacy of Lignocaine in Gel and Spray form during Buccal Infiltration Anesthesia in Children: A Randomized Clinical Trial. J Contemp Dent Pract 2014;15(6):750-754.

Source of support: Nil

Conflict of interest: None

${ }^{1}$ Former Postgraduate Student, ${ }^{2}$ Additional Professor ${ }^{3}$ Associate Professor, ${ }^{4}$ Professor and Head

1,2,4 Department of Pedodontics and Preventive Dentistry Manipal College of Dental Sciences, Manipal University Mangalore, Karnataka, India

${ }^{3}$ Department of Public Health Dentistry, Manipal College of Dental Sciences, Manipal University, Mangalore, Karnataka India

Corresponding Author: BS Suprabha, Additional Professor Department of Pedodontics and Preventive Dentistry, 'Shreyas' 15/17/940-13, 5th Cross Road, Shivabagh, Kadri, Mangalore 575002, Karnataka, India, Phone: 091-0824-2428716, Fax: 091-0824-2422653, e-mail:suprabha.bhat@manipal.edu

\section{INTRODUCTION}

Children are often reluctant to undergo dental treatment due to fear and anxiety associated with pain. ${ }^{1}$ Local anesthesia is one of the effective pain control methods. However, ironically, the means of administering local anesthesia, namely injection, can itself cause fear and anxiety. The pain and discomfort resulting from injection can be minimized by using a variety of techniques. These include appropriate behavior management techniques, altering the $\mathrm{pH}$ and temperature of the anesthetic solution and injecting the solution at reduced rate. Another effective method is to anesthetize the surface mucosa before needle insertion. The methods by which anesthesia of surface tissues can be achieved include refrigeration, transcutaneous electronic nerve stimulation and topical anesthesia. $^{2}$

Topical anesthesia is achieved by the pharmacologic action of the anesthetic agent on the surface tissues, when applied to it. Topical anesthetics function by blocking signal transmission in the terminal fibers of sensory nerves. ${ }^{3}$ They are effective only on surface tissues upto 2 to $3 \mathrm{~mm}$ depth. ${ }^{4}$ Topical anesthetics have both psychological and pharmacological effects. Subjects who are informed that they are to receive a topical anesthetic for comfort during injection of local anesthetic anticipate less pain during injection than those not offered such counselling. ${ }^{5,6}$ The literature reports mixed results regarding the pharmacological efficacy of topical anesthetics. ${ }^{6}$ There is evidence from numerous placebo controlled double blind clinical trials that topical anesthetics have pharmacological effect. Animal and human studies have shown that the effect of local anesthetics is concentration dependent. The efficacy is better in nonkeratinized mucosa than keratinized mucosa. In addition, topical anesthetics mask pain of superficial infiltration injection better than for deep regional block injections. ${ }^{2,7}$

Lignocaine and benzocaine are the most commonly used topical anesthetics among the various amide and ester agents. ${ }^{3}$ When used as a single agent, lignocaine is effective between concentrations of 5 to $20 \%$ and theresponse is dose dependent. ${ }^{2}$ Topical lignocaine has very low incidence of allergic reactions as compared to esters such as benzocaine. ${ }^{4}$ 
The efficacy of topical anesthetics depends on various factors such as the drug used, concentration, $\mathrm{pH}$, site of injection, additives, formulation and duration of application. ${ }^{7}$ Topical anesthetics are commercially available as aerosols, ointments, gels, lozenges, tablets, pastes, powders, solutions and impregnated patches. The concentration of the agent varies depending on the formulation. For example, topical sprays would require a higher concentration of anesthetic agent than patches which in turn would affect the efficacy. ${ }^{2}$ The formulation of the drug may also affect the ability to localize the drug at the site of injection, controlled release of the drug, thus prolonging the action. ${ }^{7}$ Lignocaine spray and gel are two commonly used forms of topical anesthetic agent. Lack of studies comparing the efficacy of these two formulations in the literature prompted us to conduct this study which would enable the clinician to choose between an anesthetic spray and gel, for use in children.

The purpose of our study was to evaluate and compare the efficacy of lignocaine in two different formulations in reducing pain caused by needle insertion during administration of local anesthesia.

\section{AIMS AND OBJECTIVES}

To compare the efficacy of $15 \%$ lignocaine spray and $8 \%$ lignocaine gel as a topical anesthetic, in reducing pain, during buccal infiltration in children.

\section{MATERIALS AND METHODS}

\section{Study Design and Sample Size}

The present study was a randomized clinical trial. Assuming 50\% difference in the pain threshold between Lignocaine gel and Lignocaine spray groups, the sample size for this study was calculated to be 21 in each group at $95 \%$ confidence interval and a power of $90 \%$ using Stata/ SE 10.0 (Stata Corp, USA) software.

\section{Inclusion Criteria}

All patients attending a pediatric dental department for routine dental treatment, over a period of 2 months, were screened for inclusion criteria.

1. Patients requiring restorative procedures/extraction/ pulp therapy of primary/permanent teeth in the maxillary arch, which need administration of buccal infiltration anesthesia.

2. Patients aged between 7 and 12 years.

3. Patients who exhibited cooperative behavior (Frankl scale 3 or 4 ).

4. Patients who demonstrated competency in using the Wong-Baker FACES pain rating scale.

\section{Exclusion Criteria}

1. Patients with known drug allergies toward topical anesthetic agents.

2. Patients with pre-existing debilitating systemic diseases.

3. Patients whose parents did not give informed consent to participate in the study.

Thus, a total of 42 patients were selected for the study. Ethical approval: The study was conducted after obtaining an approval from the Institutional Ethics Committee. Parents of the patients were informed about the requirements and the complications of the procedure through a patient information sheet following which their informed consent was obtained.

\section{Methodology}

The candidates were randomly allocated to two groups:

Group A: 8\% Lignocaine gel (Precaine ${ }^{\circledR}$, Pascal Inc., USA) with 21 subjects.

Group B: 15\% Lignocaine spray (Nummit ${ }^{\circledR}$, ICPA, India) with 21 subjects.

The lignocaine spray used in this study consisted of Lignocaine hydrochloride, propylene glycol, menthol, clove oil and propellant. The lignocaine gel used in this study consisted of $0.8 \%$ dibucaine along with lignocaine in base form. Each subject participating in the study was administered only one type of topical anesthetic agent. The randomization was done using chit pick box method. A box containing 21 chits labelled 'group- $\mathrm{A}$ ' and 21 chits labelled 'group- $\mathrm{B}$ ' was prepared and kept ready at the start of the study. The chit was picked by the patient from the box, and was assigned to the group according to the chit picked.

The application of the topical anesthetic agents was done by the first investigator. The reason for the use of the topical anesthetic was communicated to the patient using appropriate euphemisms. The application site was dried with sterile cotton gauze before application of either of the topical anesthetic. In group A, approximately $0.5 \mathrm{gm}$ of $8 \%$ lignocaine gel was applied to the height of the mucobuccal fold, using a sterile cotton applicator tip. The applicator tip was inserted into the gel container and rotated clockwise 3 times to standardize the amount of drug applied. The gel was applied with moderate pressure with rubbing motion for 30 seconds to increase the depth of penetration. In group B, lignocaine spray which expels $7.5 \mathrm{mg}$ lignocaine per spray was sprayed on the region to which buccal infiltration was to be administered, from a distance of 1 to $2 \mathrm{~cm}$. In both the groups, the mucosa was wiped with cotton gauze, 3 minutes after the application of the topical anesthetic material. 
The administration of the buccal infiltration was done by the second investigator who was blinded to the topical anesthetic agent used. The buccal infiltration was administered using a 30 gauge short needle syringe (Dispovan, HMD Ltd., India). $1 \mathrm{ml}$ of $\%$ lignocaine with 1:200,000 adrenaline (Astra Zeneca Pharma, India Ltd.) was injected. Standard technique ${ }^{4,8}$ for buccal infiltration anesthesia was followed keeping the rate of injection as $0.8 \mathrm{ml} / \mathrm{min}$. In order to maintain consistency, the second investigator gave standard set of verbal instructions to the patient prior to administering the local infiltration anesthesia, using appropriate euphemisms, verbal reinforcement distraction techniques. During injection, care was taken to keep the needle out of directsight of the child. The lip/cheek was stretched to make the tissue taut. Holding the syringe parallel to the long axis of the tooth, with needle oriented such that the bevel faces the bone, the needle was inserted at the height of the mucobuccal fold over the target tooth. The depth of penetration was 2 to $3 \mathrm{~mm}$. The needle was penetrated slowly into the tissue along with low speed continuous injection, so that anesthesia of the tissue precedes the penetration of the needle. This was done to minimize pain during injection. ${ }^{9}$ Distraction using verbal method was done during needle penetration. In addition, verbal reinforcements were given for cooperative behavior during the course of infiltration. Standardization of technique was achieved by the second investigator by performing the same procedure on several patients under supervision before the start of the study.

The scoring was done immediately after administration of the local anesthetic. Each child quantified the pain perceived during the injection using a Wong-Baker faces pain rating scale (WBFPRS). ${ }^{10}$ WBFPRS is an ordinal faces scale which has happy face in lower anchor and face with tears in upper anchor. A third operator, who was also blinded to the topical anesthetic agent used, assessed the pain using the faces legs activity cry and consolability (FLACC) pain scale ${ }^{11}$ during administration of the local anesthetic. This simple scale consists of five categories, each being scored from 0 to 2 , resulting in a total score ranging from 0 to 10 . The third operator was calibrated using ten patients who required treatment under local anesthesia but not involved in the study.

\section{Statistical Analysis}

Age, gender, FLACC scores and Wong Baker Faces Pain Scores were recorded, and descriptive statistics was calculated for each variable. The mean value and standard deviation were calculated from the reported pain levels for each category. Independent t-test was utilized to compare the patients' response to the two agents. Pearson's chi-square test was utilized to compare the behavioral response of the patients in both the groups, as evaluated by FLACC scale. Statistical analysis was performed using SPSS version 16 (SPSS Inc., Chicago, IL, USA). The statistical significance level was set at $\mathrm{p}<0.05$.

\section{RESULTS}

A total of 23 males and 19 females patients with an average age of $9.67 \pm 1.79$ participated in the study. Among the teeth anesthetized, 37 were posterior and 5 were anterior teeth. No irritation to mucosa or other adverse effects were reported. Pearson chi-square test revealed that there was no significant effect of factors like age $(p=0.5)$ and gender $(p=0.08)$ on the pain response, in either groups. Pearson's chi-square test also demonstrated that there was no significant difference in the FLACC scores, when either of the two agents were used $(p=0.54)$ (Table 1$)$. As per the FLACC scores none of the study subjects had severe pain/discomfort and only one subject was assessed to have moderate pain. In the self-report of pain also, as done by WBFPRS scale most of the study subjects reported little or no pain. ${ }^{12}(28.6 \%)$ scored 'no hurt', 16 (38.1\%) scored 'hurts just a little bit', 9 (21.4\%) scored 'hurts little more while 4 (9.5\%) scored 'hurts even more' and only one subject $(2.4 \%)$ reported severe pain ('hurts as much as you can imagine'). Independent t-test demonstrated that there was no significant difference in patient response (Wong-Bakers Faces Pain Score) when either of the two agents were used $(p=0.07)$ (Table 2$)$.

\section{DISCUSSION}

Quantifying pain remains one of the most challenging tasks for researchers, especially when working with children. It is a highly individualized experience and difficult to measure accurately. Methods to measure pain include self-reports, biological measures such as heart rate, and behavioral measures, such as crying, movement, or agitation. Generally, patient's self-report is considered to be a reliable and effective way of measuring pain. Among children, faces pain scales should be preferred as they are simple to use and less abstract than visual analog scale. ${ }^{12}$ Face scales do not require the child to translate

Table 1: Comparison of FLACC scores using Pearson's chi-square test

\begin{tabular}{lllll}
\hline Groups & $\begin{array}{l}\text { Relaxed and } \\
\text { comfortable } \\
(0)\end{array}$ & $\begin{array}{l}\text { Mild } \\
\text { discomfort } \\
(1-3)\end{array}$ & $\begin{array}{l}\text { Moderate } \\
\text { pain (4-6) }\end{array}$ & $\begin{array}{l}\text { Severe } \\
\text { pain/ } \\
\text { discomfort/ } \\
\text { both (7-10) }\end{array}$ \\
\cline { 2 - 5 } & $n(\%)$ & $n(\%)$ & $n(\%)$ & $n(\%)$ \\
\hline A (Gel) & $9(45)$ & $11(52.4)$ & $1(100)$ & $0(0)$ \\
B (Spray) & $11(55)$ & $10(47.6)$ & $0(0)$ & $0(0)$ \\
\hline Total & $20(100)$ & $21(100)$ & $1(100)$ & $0(0)$ \\
\hline
\end{tabular}

$\chi^{2}=1.25$ (df 2), $p=0.54$ 
Table 2: Wong-Baker faces pain score interpretation using independent t-test

\begin{tabular}{llllllll}
\hline Groups & $N$ & Mean & Standard deviation & Standard error mean & $t$-value & $p$-value & $C l$ \\
\hline A (Gel) & 21 & 1.52 & 1.25 & 0.27 & 1.85 & 0.07 & $1.3-0.06$ \\
B (Spray) & 21 & 0.90 & 0.89 & 0.19 & & & \\
\hline
\end{tabular}

Cl: Confidence interval

pain experience into a numerical value; however, they do require a child to discriminate among different levels of pain depicted in pictures. Since, the school aged children who participated in this study, are in the stage of concrete operations, it is an excellent measure to measure selfreported pain in this age group. ${ }^{13}$

The Wong-Baker faces pain rating scale, used in this study, is simple to use, understood easily and has been effectively used in patients 3 years of age and older. ${ }^{10}$ It is inexpensive to re-produce and has good acceptability among children, parents and clinicians as compared to other faces pain scales. However children's self-report of pain may be influenced by developmental, cognitive and situational issues. A major concern with the WBFPRS is the confounding of emotion with pain intensity during self-report. Children who do not cry with intense pain, especially older boys, may be reluctant to pick the face scored 5 because it shows tears. ${ }^{12}$ In addition, a child's dental anxiety may lead him or her to evaluate their pain, inaccurately. ${ }^{13}$ Hence, observational and/or physiologic measures should be used in conjunction with self-report measures. ${ }^{12}$ Therefore, to overcome this obstacle, another pain rating scale. The face legs activity cry and consolability (FLACC) scale, was used as an additional tool to assess pain in this study. Use of both the scales confirmed that both lignocaine gel and spray have equivocal effect in reducing pain during needle penetration.

Double blinding used in this study eliminated any bias that may influence the technique of administration of local anesthetic or the pain assessment. Most of the previous studies, evaluating the effects of different topical anesthetic agents, have used a split-mouth design, using all the test materials, in the same patient. ${ }^{7}$ However, this might lead to a bias, as the patient's previous exposure to injection may influence the self-report of the pain. Though the variability in individual pain response is addressed in a split mouth design, the injection order can influence patient perception of pain with the second injection being perceived as more painful than the first. ${ }^{5}$ Bagesund and Tabrizi ${ }^{9}$ found that good pain control could reduce the patients' anxiety level at the second appointment and hence this can affect the pain scores. To overcome this bias, a split mouth design was not used in this study and only a single agent was applied in every patient. We did not use a control group with placebo in this trial as there is good evidence of pharmacologic benefits of topical anesthesia in minimising pain due to needle penetration during injection of local anesthetic. ${ }^{2}$ The use of topical anesthetic prior to the injection of a local anesthetic to reduce discomfort associated with needle penetration has been recommended by American Academy of Pediatric Dentistry. ${ }^{4}$

The results of this study indicate that there was no difference between the two formulations tested in reducing pain during buccal infiltration. Low pain scores in this study may be attributed to verbal suggestion; behavior management techniques used resulting in psychological benefits, use of standard technique to inject the local anesthetic, in addition to pharmacologic effects of the topical anesthetic. The psychological perception of pain can be altered by culture, personal history (previous experiences), personality, emotion and a cognitive ability, ${ }_{1}^{14}$ which however were not considered in this study and is a limitation. Many subjects in the study reported mild to moderate pain which indicates that application of topical anesthetics did not result in totally pain free buccal infiltration anesthesia procedure in the maxillary arch. Time of application of the topical anesthetic was 3 minutes. A comparison of positive and negative response studies of topical anesthetic application prior to needle insertion showed a minimum 2 minute application is necessary for positive response. Longer application time can decrease the discomfort during injection. ${ }^{7}$ In this study, pain assessment was done after injecting the solution which may confound the results, according to some authors. ${ }^{14,15}$ Hence, in needle penetration studies pain assessment is donesoon after the needle prick and no solution is injected. However, injection studies have the advantage of the procedure being carried out in a real clinical situation, which makes the results more reliable. Assessment of pain after injection of local anesthetic may result in higher pain score. ${ }^{16}$ Hence, care was taken in our study to standardize the procedure of injection, such as wiping the mucosa dry before topical application to maximize the effect of the topical anesthetic, application time of 3 minutes, use of smaller gauge needle, slow rate of injection, verbal suggestion and behavior management techniques. This standardization was done to minimise pain as well as variation between the groups.

Topical anesthetic spray and gel have both advantages and disadvantages. The advantages of topical gels include better localization of drug in comparison with ointments and solutions, better control over systemic drug absorption, greater bioavailability and reduction in 
dosage. ${ }^{17}$ In addition a flavored gel is better accepted by children. ${ }^{18}$ However, gels get diluted in the mouth with time, resulting in is difficulty in maintaining prolonged mucosal contact resulting in inadequate anesthesia. ${ }^{15}$ The Precaine gel used in the study contains dibucaine in small concentrations $(0.8 \%)$ which is also a local anesthetic. Dibucaine is used in the field of dermatology to treat pain and itching in minor burns, sunburn, hemorrhoids and insect bites. ${ }^{18}$ The use of dibucaine as topical anesthetic is rarely documented in dentistry. Yamamura et al ${ }^{19}$ successfully used dibucaine containing adhesive films for control of pain due to oral ulcers. The advantage of dibucaine is prolonged anesthetic effect. The lignocaine present in the gel results in faster onset (35-40 seconds) and the dibucaine prolongs the anesthetic effect (3040 minutes). ${ }^{18}$ Compound topical anesthetics increase the risk of systemic toxicity but when used cautiously, with predetermined dosage, can be safe and effective. ${ }^{4}$ Deepika et $\mathrm{al}^{18}$ reported lower mean pain scores with lignocaine-dibucaine gel in comparison with benzocaine gel although statistically the difference was not significant. Topical anesthetic sprays have greater concentration of local anesthetic and are absorbed rapidly across the mucous membrane, thus providing effective anesthesia. Unmetered sprays have potential for systemic toxicity and hence it is recommended that metered spray with disposable nozzles be used. ${ }^{3}$ Nummit spray used in this study fulfils this recommendation. The spray contains lignocaine hydrochloride in water-oil based emulsion, which increases tissue penetration and access into the nerve cell. ${ }^{7}$ However, there is difficulty in confining the effect of a drug to a small area, decreased bioadhesion thus decreasing its efficacy. ${ }^{20}$ In addition, spray has been rated asunpleasant and also may cause difficulty in swallowing in some individuals. ${ }^{21}$ Despite these disadvantages, lignocaine gel and spray are commonly used in clinical practice than adhesive patches or disks as they increase the cost of the treatment phenomenally. ${ }^{17}$

It can be inferred from the results that, as long as standard techniques of local anesthesia administration are followed, it remains to the discretion of the clinician to choose the formulation of lignocaine as a topical anesthetic agent when dealing with a child patient. The clinician may consider patient preference which however was not evaluated in this study.

\section{CONCLUSION}

There is no significant difference between the efficacy of $15 \%$ lignocaine spray and $8 \%$ lignocaine gel as a topical anesthetic in controlling pain during buccal infiltration anesthesia, in children.

\section{REFERENCES}

1. Hutchins HS Jr, Young FA, Lackland DT, Fishburne CP. The effectiveness of topical anesthesia and vibration in alleviating the pain of oral injections. Anesth Prog 1997 Summer;44(3): 87-89.

2. Meechan JG. Effective topical anesthetic agents and techniques. Dent Clin North Am 2002 Oct;46(4):759-766.

3. Malamed SF. Handbook of local anesthesia. 5th ed. St. Louis: Mosby; 2004. p. 119-121.

4. American Academy on Pediatric Dentistry Council on Clinical Affairs. Guideline on appropriate use of local anesthesia for pediatric dental patients. Pediatr Dent 2008-2009;30(7 Suppl):134-139.

5. Martin MD, Ramsey DS, Whitney C, Fiset L, Weinstein P. Topical anesthesia: differentiating the pharmacological and psychological contributions to efficacy. Anesth Prog 1994; 41(2):40-47.

6. PollackS. Pain control by suggestion. J Oral Med 1966 Apr;21(2): 89-95.

7. Meechan JG. Intraoral topical anesthesia. Periodontology 2000, 2008 Feb;46(1):56-79.

8. Malamed SF. Basic injection technique. In: Handbook of Local Anesthesia. 5th ed. St. Louis, Mo: Mosby; 2004;159-169.

9. Bågesund M1, Tabrizi P. Lidocaine $20 \%$ patch vs lidocaine $5 \%$ gel for topical anesthesia of oral mucosa. Int J Paediatr Dent 2008;18(6):452-460.

10. Wong DL, Baker CM. Pain in children: comparison of assessment scales. Pediatr Nurs 1988;14(1):9-17.

11. Merkel SI, Voepel-Lewis T, Shayevitz JR, Malviya S. The FLACC: a behavioral scale for scoring postoperative pain in young children. Pediatric Nurs 1997;23(3):293-297.

12. Tomlinson D, von Baeyer CL, Stinson JN, Sung L. A systematic review of faces scales for the self-report of pain intensity in children. Pediatrics 2010 Nov;126(5):e1168-1198.

13. Garra G1, Singer AJ, Taira BR, Chohan J, Cardoz H, Chisena E, Thode HC Jr. Validation of the Wong-Baker FACES Pain Rating Scale in pediatric emergency department patients. Acad Emerg Med 2010 Jan;17(1):50-54.

14. Rosivack RG, Koenigsberg SR, Maxwell KC. An analysis of the effectiveness of two topical anesthetics. Anesth Prog 1990 Nov-Dec;37(6):290-292.

15. Hersh EV, Houpt MI, Cooper SA, Feldman RS, Wolff MS, Levin LM. Analgesic efficacy and safety of an intraoral lidocaine patch. J Am Dent Assoc 1996 Nov;127(11):1626-1634.

16. Carr MP, Horton JE. Evaluation of a transoral delivery system for topical anesthesia. J Am Dent Assoc 2001 Dec;132(12): 1714-1719.

17. Satya Bhushan NV, Nayak RN. A comparison of the efficacy of topical application of lignocaine hydrochloride $5 \%$ gel and bupivacaine hydrochloride $5 \%$ gel for extraction of teeth. J Maxillofac Oral Surg 2010 Jun;9(2):119-126.

18. Deepika A, Rao CR, Vinay C, Uloopi KS, Rao VV. Effectiveness of two flavored topical anesthetic agents in reducing injection pain in children: a comparative study. J ClinPediatr Dent 2012 Fall;37(1):15-18.

19. Yamamura K, Yotsuyanagi $T$, Okamoto T, Nabeshima T. Pain relief of oral ulcer by dibucaine-film. Pain 1999;83(3):625-626.

20. Tulga F, Mutlu Z. Four types of topical anesthetic agents: evaluation of clinical effectiveness. J ClinPediatr Dent 1999 Spring;23(3):217-220.

21. Haasio J, Jokinen T, Numminen M, Rosenberg PH. Topical anesthesia of gingival mucosa by $5 \%$ eutectic mixture of lignocaine and prilocaine or by $10 \%$ lignocaine spray. Br J Oral Maxillofac Surg 1990 Apr;28(2):99-101. 